\title{
Modeling and Simulation of SSPC based on Dymola Software and Modelica Language
}

\author{
Yufeng Wang ${ }^{1} \quad$ Yufei Tao $^{1}$ Qinzhou Lin ${ }^{1} \quad$ Weilin $\mathrm{Li}^{1}$ \\ ${ }^{1}$ College of automation, Northwestern Polytechnical University, China,wyfnwpu@mail.nwpu.edu.cn, \\ taoyufei0412@163.com,shforest@hotmail.com, liweilin907@126.com
}

\begin{abstract}
Solid State Power Controller (SSPC) is one of the key components of aircraft power distribution system. It is a switch device composed of solid-state semiconductor devices, which is used to switch on/off the circuit and realize the control and protection of power distribution bus. It is one of the important components of aircraft automatic power distribution technology. Compared with traditional mechanical switches, the SSPC does not cause mechanical wear, has a low failure rate, and has high reliability, which is especially suitable for aviation applications. Compared with smart contactors, SSPC is small in size and high in integration, meeting the streamlined requirements of advanced aircraft electrical systems for power distribution devices. The Dymola simulation software based on Modelica language has the advantages of simple operation, high integration, and convenient for collaborative simulation. This paper first introduced the basic principle of SSPC, then combined with Modelica language and Dymola simulation software to model and verify the function of current limiting, overcurrent protection and short circuit protection of SSPC, numerical simulation results were also provided.
\end{abstract}

Keywords:SSPC, Dymola modeling, Modelica language.

\section{Introduction}

Avionics, weapons, and flight control systems are becoming more and more complete with the rapid development of aeronautical technology, aircraft performance has been greatly improved, and more and more airborne electrical equipment has been used. The aircraft distributed power distribution system has become the future development direction. Traditional mechanical switches can no longer meet the needs of circuit protection. Solid-state power controllers that can be integrated in power distribution systems are an important part of distributed power distribution systems.

The SSPC is an intelligent switching device composed of semiconductor devices. As the core component of Secondary Power Distribution Assembly, it is used to switch on and off the circuit, realize circuit protection, receive the control signal of the preceding computer and upload the working status to it [1]. Its function is similar to the traditional combination of circuit breakers and relays in series or other control protectors. It is used to replace the relay's conversion function and the protection function of circuit breaker, the functional equivalent diagram of SSPC is shown in Figure 1. It is much better than these traditional devices in performance and function. SSPC uses solid-state semiconductor devices as switching devices, which can quickly turn on and off the circuit with extremely short delay. Because solid-state switching devices have no contacts or moving parts. the SSPC does not cause mechanical wear, has a low failure rate, and has high reliability, which is especially suitable for aviation applications [2]. Compared with smart contactors, SSPC is small in size and high in integration, which meets the requirements for simplified power distribution devices of advanced aircraft electrical systems [3].

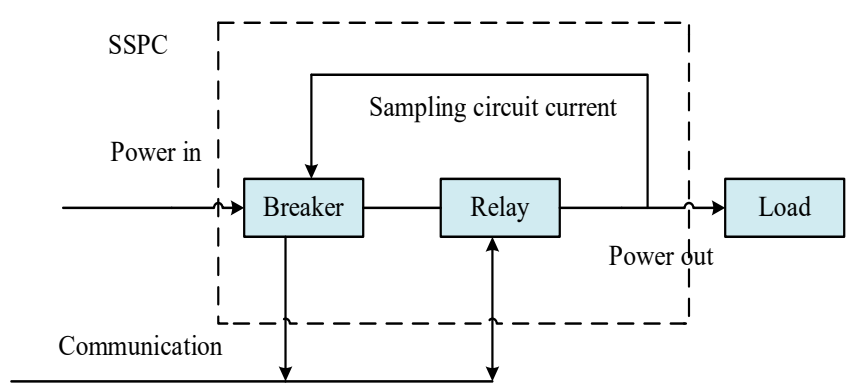

Figure 1. Functional equivalent diagram of SSPC

Dymola simulation software is suitable for modeling various physical systems. It supports a hierarchical model structure, a truly reusable component library, wiring terminals and composite acasual connections. The model library can be applied in various engineering fields [4-5].

Dymola uses a new object-oriented and equationbased modeling approach. The automatic processing of equations replaces the manual conversion of equations to block diagrams in the traditional sense. Other highlights of Dymola are as follows:

- Handle large, complex, multi-engineering models.

- $\quad$ Faster modeling by building graphical models.

- Faster simulation --symbol preprocessing.

- Open user-defined model elements.

- Open interface to other programs. 
- $\quad 3 \mathrm{D}$ animation effect.

- Real-time simulation.

In this paper, the Dymola simulation software based on Modelica language is selected to build and simulate a $28 \mathrm{~V}$ DC SSPC model, including overvoltage protection, overcurrent protection, short circuit protection and other functions.

\section{SSPC Principle Analysis}

As can be seen from Figure 2, the SSPC is mainly composed of logical control, MOSFET driver, power switch tube, sampling resistor, overvoltage protection module, overcurrent protection module and so on. The logical control module uses intelligent chips (or simple D flip-flops and NAND gates) to judge the voltage and current signals, and uses the result as the input of the MOSFET driving circuit to drive the MOSFET. The sampling resistor connected between the switch tube and the airborne test equipment is used to sample the current and voltage.

According to the above analysis of SSPC principle, it can be seen that it is different from the conventional thermal protection method used to achieve circuit protection in the power distribution system. Instead, it detects the current and voltage to drive the MOSFET. The performance is far superior to thermal protection devices, and its damage characteristics can be designed to be lower than the damage characteristics of on-board test equipment, achieving a more perfect protection function for the distribution network.

On the other hand, due to the fast response speed, SSPC can quickly cut off the power supply line when a short-circuit fault occurs, preventing a short-circuit from causing a sharp drop in the grid voltage and causing power interruption. Combined with the reverse-current diode, it can prevent the voltage change of the power supply bus bar and realize the uninterrupted power supply of the DC power supply.

\section{Modeling various parts of SSPC}

\subsection{Overvoltage protection module}

The overvoltage protection mainly completes the overvoltage protection function. Here, the operating point voltage of the overvoltage protection is set to $28 *$ $2 \mathrm{~V}$. When the output voltage of the channel exceeds the operating point voltage, this channel is protected and the DC contactor it controls is disconnected. When the output voltage returns to normal, the DC contactor should be switched on again.

According to the design requirements of the overvoltage protection module, a model of the overvoltage protection module is established, as shown in Figure 3.

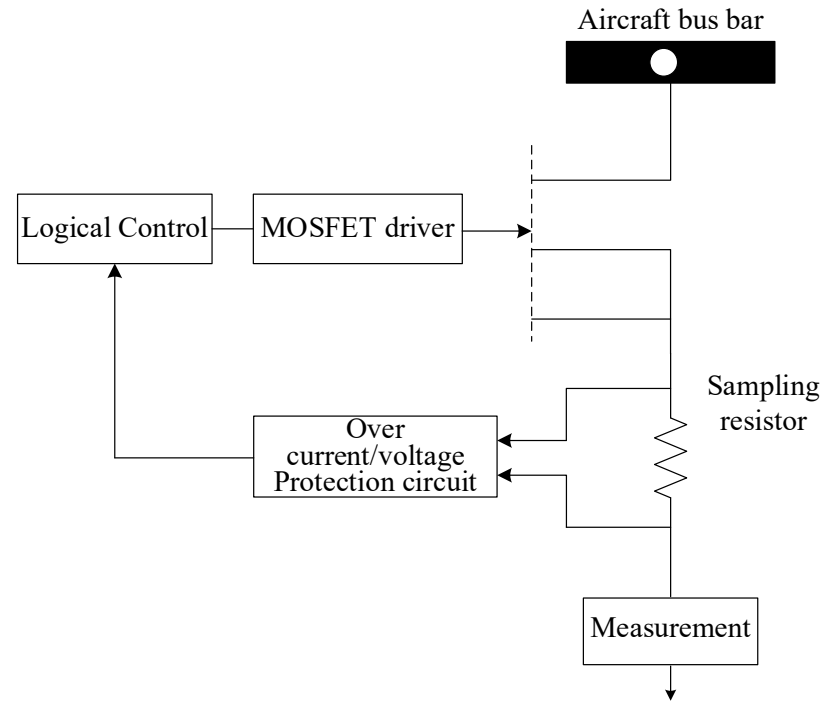

Figure 2. SSPC principle block diagram

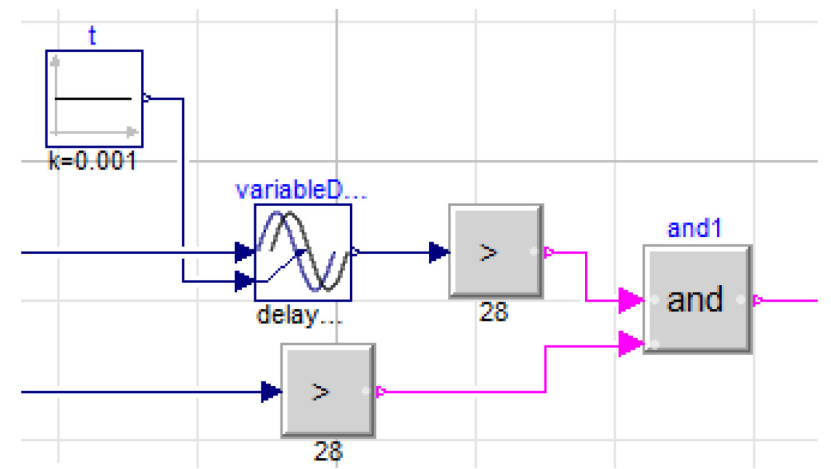

Figure 3. Overvoltage protection module

\subsection{Overcurrent protection module}

The difficulty of SSPC is the protection processing when the line is overloaded. This paper uses the $I^{2} t$ protection curve to protect the line from overload. This is because the power loss of the line is equal to the product of the line resistance and the square of the current, and the temperature of the line is determined by the time the line consumes. For power distribution lines, $\mathrm{I}^{2} \mathrm{t}$ is a constant value and exceeding this value may cause damage to the power distribution lines. Therefore, SSPC uses $I^{2} t$ protection curves for the protection of key lines [6].

Inverse time-limit overcurrent protection of transmission lines generally adopts the following three standard inverse time-limit characteristic equation:

(1) General inverse time limit

$$
\mathrm{t}=\frac{0.014 T_{p}}{\left(I / I_{P}\right)^{0.02}-1}
$$

(2) Very inverse time limit

$$
\mathrm{t}=\frac{1.35 T_{p}}{I / I_{P}-1}
$$

(3) Extreme inverse time limit 


$$
\mathrm{t}=\frac{8 T_{p}}{\left(I / I_{P}\right)^{2}-1}
$$

In general, $\mathrm{t}$ is the operating time; $T_{p}$ is the time constant; $I$ is the effective value of the rated current; $I$ is the effective value of the current at the time of the fault and $I_{p}$ is the effective value of the rated current. In this paper, the very inverse time limit method is used, and its inverse time protection curve is shown in Figure 4.

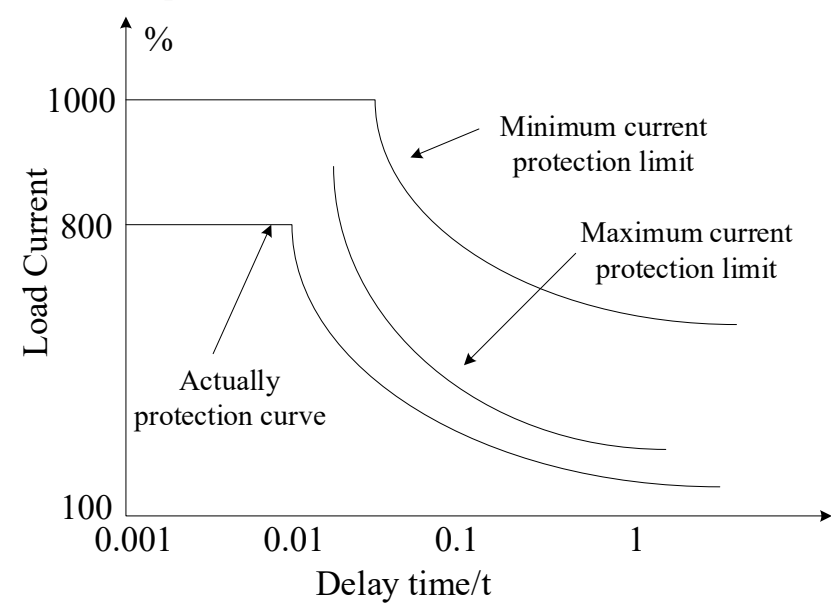

Figure 4. Typical inverse time limit curve of SSPC

The overcurrent protection mainly completes the overcurrent protection function. When the control protector detects the overcurrent fault at the output, the connection contactor of the local system and the DC contactor of the channel should be disconnected according to the anti-delay protection characteristic curve.

According to the requirements of overcurrent protection function, when the current is greater than the typical value in the overcurrent protection delay curve, the module needs to realize the reverse delay protection and the state output retention module mentioned above. The module construction is shown in Figure 5.

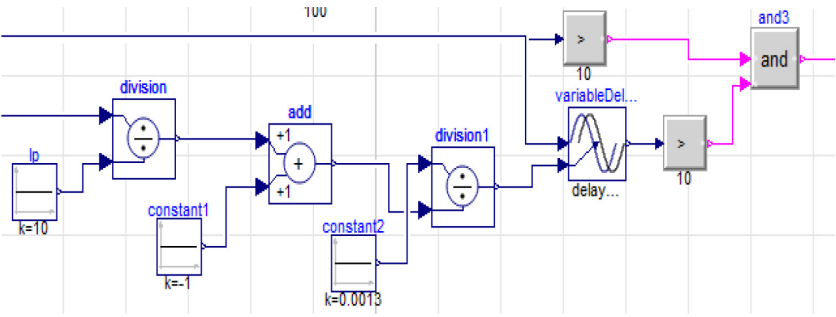

Figure 5. Overcurrent protection model

Because the overcurrent protection cannot be restored automatically, the output holding module is required to hold its output. When outputting a shutdown signal, the shutdown signal should be kept from disappearing. Therefore, an S-R trigger is added after the fault signal to achieve this function.

\subsection{Short circuit protection module}

The instantaneous value of the short-circuit current can reach tens or even hundreds of times of the rated current. Therefore, if the ordinary reverse delay protection strategy is used, it will cause irreparable damage to the main circuit switch tube and the diode, and even damage the main circuit device by breakdown [7]. A high current surge will occur at the moment the circuit is turned on, but this current is a normal generated current with a time in the microsecond range, so it will not cause circuit damage. Delay protection measures need to abandon this state [8].

The specific requirements of the short-circuit protection function are as follows, when the control protector detects a short-circuit fault at the output terminal, it should delay the output of the short-circuit protection signal by $0.001 \mathrm{~s}$ and disconnect the contactor.

According to the requirements of the overcurrent protection function, this module needs to realize the anti-delay protection and the state output holding module described above when the current is greater than the typical value in the overcurrent protection delay curve, and the model building is shown in Figure 6.

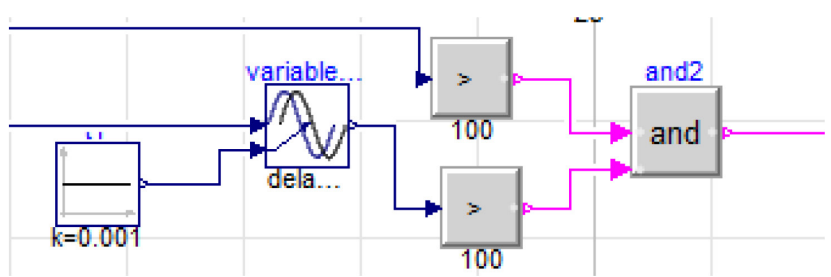

Figure 6. Short-circuit protection model

\section{System modeling and simulation}

\subsection{System modeling}

Connect the overvoltage protection, overcurrent protection, and short-circuit protection model modules that have been previously established and package them, as shown in Figure 7.

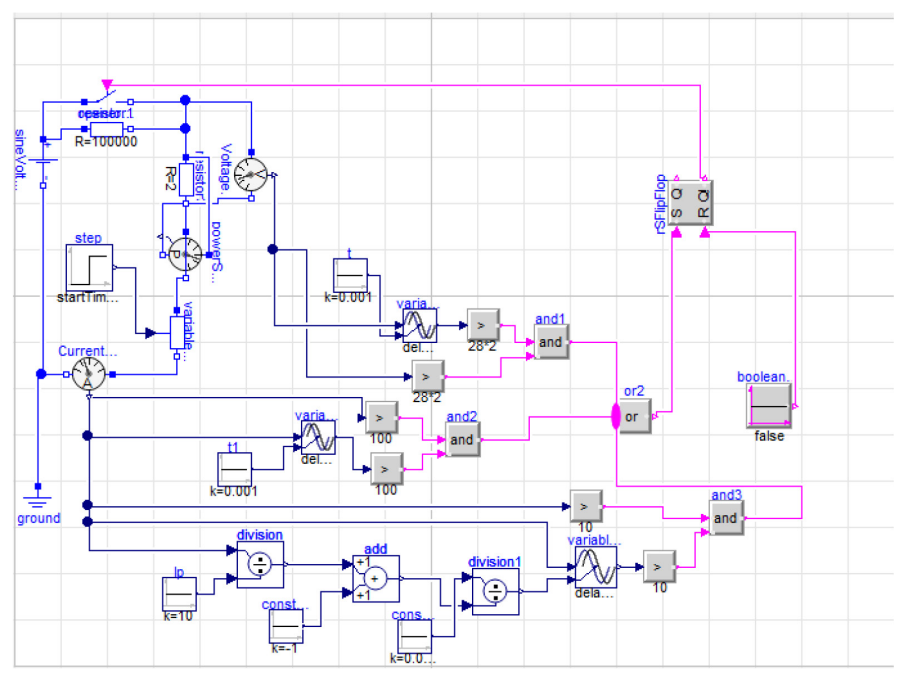

(a) Internal diagram of DC control protector model 


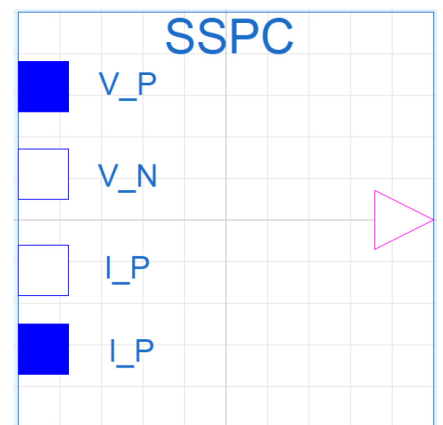

(b) SSPC model encapsulation

Figure 7. Overall protection model

\subsection{Simulation Results}

Set up the test model shown in Figure 8, input voltage $28 \mathrm{~V}$, connect to the SSPC package model, and simulate and verify the overvoltage protection, overcurrent protection and short circuit protection functions of the SSPC. In the test model, the variable resistance plays the role of partial voltage. When $0.5 \mathrm{~s}$ occurs, the resistance value of the variable resistor becomes 0 . At this point, the voltage at both ends of the load increases, resulting in overvoltage or overcurrent, thus turning off the switch to achieve protection. The simulation results are shown in figure 9 below.

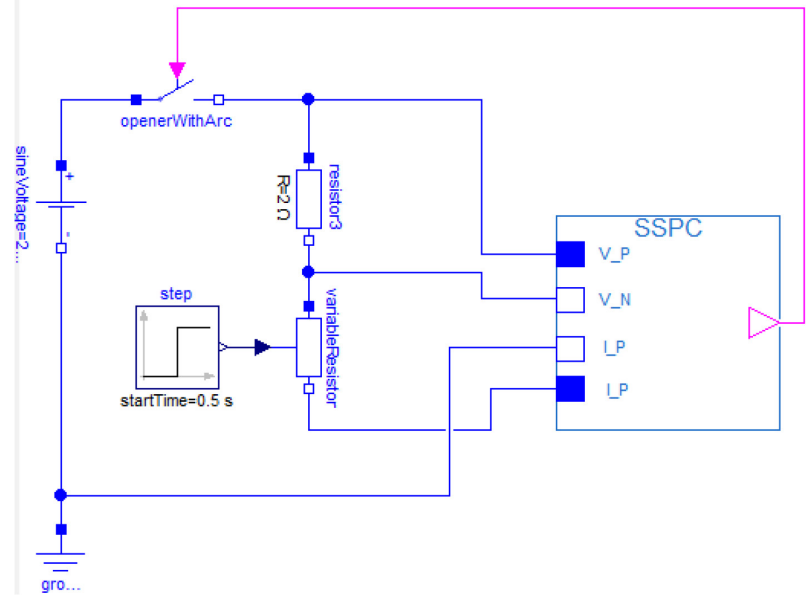

Figure 8. SSPC model test
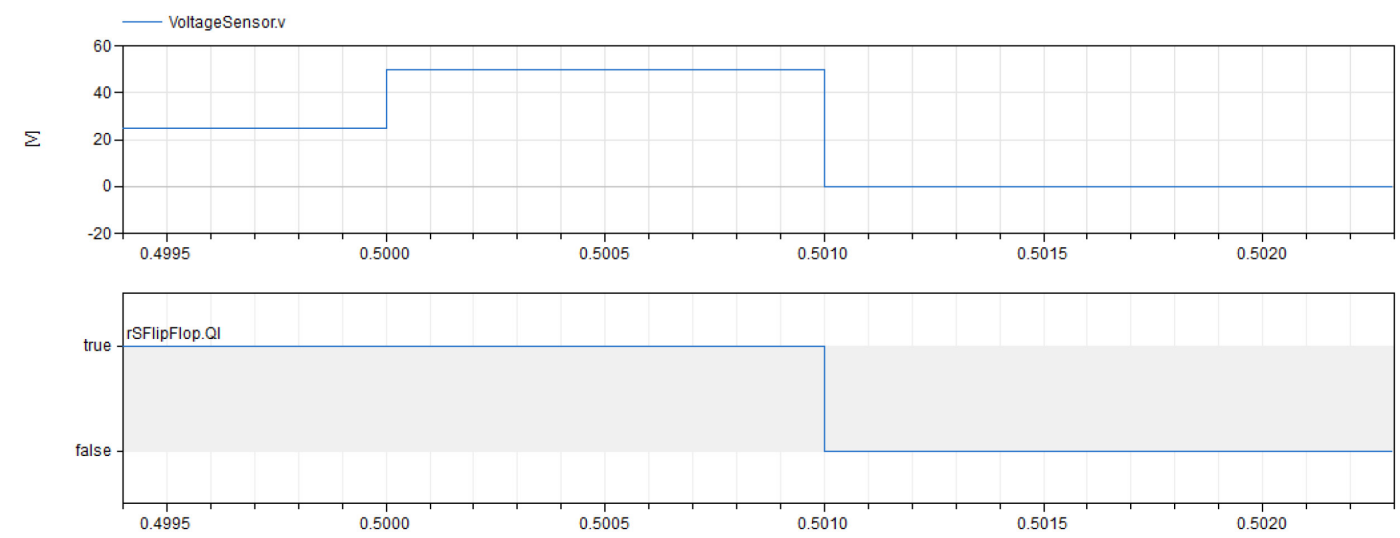

(a) Simulation results of overvoltage protection
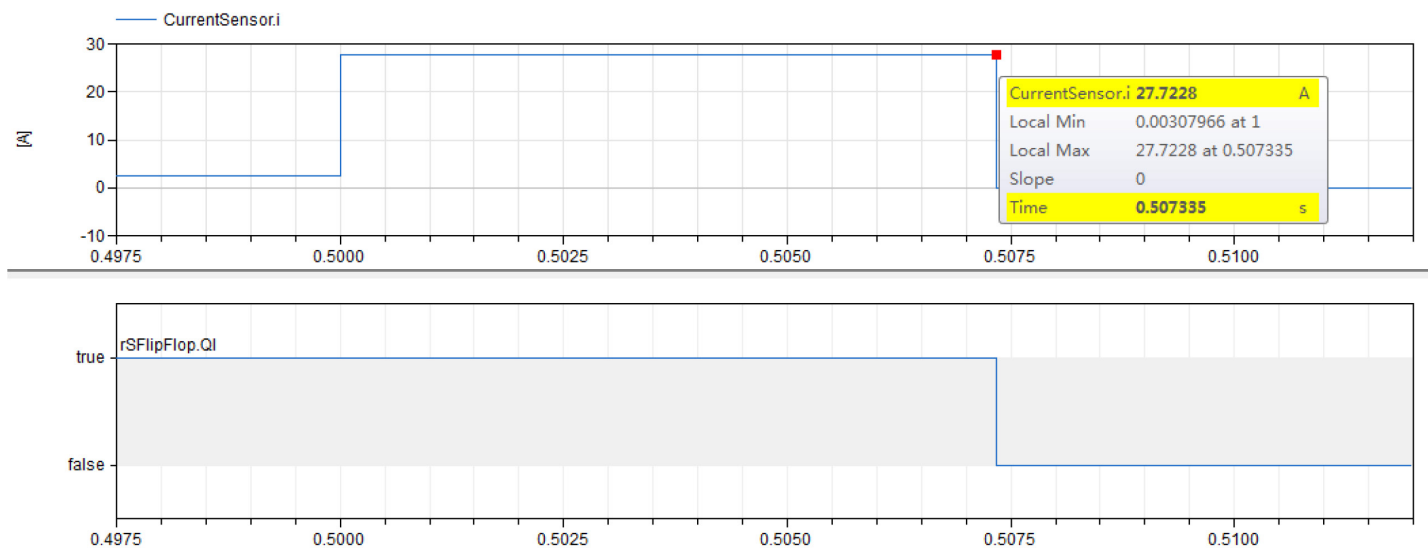

(b) Over-current inverse delay protection simulation results 

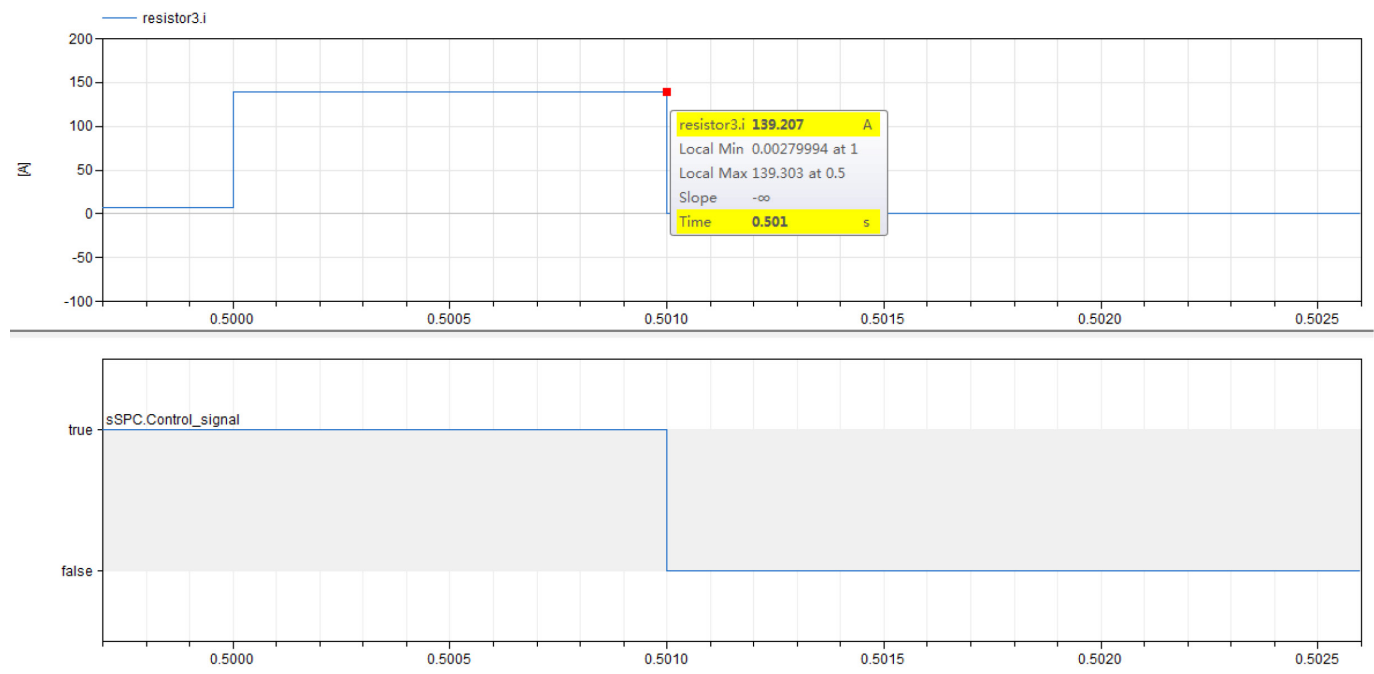

(c) Short circuit protection simulation results

Figure 9. Simulation results

From the simulation results, it can be seen that when the overvoltage protection and short circuit protection, the switch control signal delay $0.001 \mathrm{~s}$ becomes a low signal, which meets the requirements, when the load current becomes $27.7 \mathrm{~A}$, and the control signal delay is $0.0073 \mathrm{~s}$ to become a low signal, which realizes overcurrent reverse delay protection, as shown in Figure 9(b). Through the test of the building model, the correctness of the model is verified.

\section{Conclusion}

This paper builds a SSPC model based on the Dymola simulation software, and simulates its function of overvoltage protection, overcurrent protection and short-circuit protection. The correctness and accuracy of the model are verified. Compared with other simulation software, the model built in the Dymola simulation software is simpler and more convenient, which well demonstrates the characteristics of SSPC. Secondly, this model is universal and can be applied to different scenarios by modifying the corresponding parameters.

\section{References}

[1] Barrado A , Izquierdo D , Sanz M , et al. Behavioural Modeling of Solid State Power Controllers (SSPC) for Distributed Power Systems[C]// Applied Power Electronics Conference and Exposition, 2009. APEC 2009. TwentyFourth Annual IEEE. IEEE, 2009. doi:10.1109/APEC.2009.4802897.

[2] Izquierdo D, Barrado A, Raga C , et al. Protection Devices for Aircraft Electrical Power Distribution Systems: State of the Art[J]. IEEE TRANSACTIONS ON AEROSPACE AND ELECTRONIC SYSTEMS, 2011, 47(3):1538-1550. doi: 10.1109/taes.2011.5937248.

[3] Devinda A. Molligoda, Pradip Chatterjee, Chandana J. Gajanayake. Review of design and challenges of DC SSPC in more electric aircraft[C]// 2016 IEEE 2nd Annual
Southern Power Electronics Conference (SPEC). IEEE, 2016. doi: 10.1109/SPEC.2016.7846117.

[4] Machado, José, Seabra, Eurico, Soares, Filomena. A new Plant Modelling Aproach for Formal VerificationE Purposes[J]. Ifac Proceedings Volumes, 40(9):167-172. doi: 10.3182/20070723-3-pl-2917.00027.

[5] Qiong-Zhong Chen,, Yu-Feng Mo,, Guang Meng,. Dymola-based modeling of SRD in aircraft electrical system $[\mathrm{J}]$. Aerospace \& Electronic Systems IEEE Transactions on, 42(1):220-227. doi: 10.1109/TAES.2006.1603417.

[6] M. W. Stavnes, A. N. Hammoud, Assessment of Safety in Space Power Wiring Systems[J], IEEE Aerospace and Electronic Systems Magazine, 1994， 9: 21-27. doi:

[7] Ahmed M M R, Mawby P A. Design specification of a 270 V 100 A solid-state power controller suitable for aerospace applications[C]// 2009.

[8] Liu Z Z, Gayowsky T J, Fuller R J, et al. SSPC technology incorporated with thermal memory effects to achieve the fuse curve coordination[J]. 2010. doi: US7706116 B2. 